Research Paper

\title{
Developing a Selection-aided Model to Screen Cirrhotic Intrahepatic Cholangiocarcinoma for Hepatectomy
}

\author{
Qinjunjie Chen ${ }^{1^{*}}$, Fengwei $\mathrm{Li}^{1,2^{*}}$, Yuzhen Gao ${ }^{3^{*}}$, Hui Xue², Zheng $\mathrm{Li}^{1}$, Qifei Zou ${ }^{2}$, Yong Xia1 ${ }^{1}$ Kui Wang ${ }^{2}$ \\ and Feng Shen ${ }^{\circledR}$ \\ 1. Department of Hepatic Surgery IV, the Eastern Hepatobiliary Surgery Hospital, Naval Medical University (Second Military Medical University), Shanghai, \\ China. \\ 2. Department of Hepatic Surgery II, the Eastern Hepatobiliary Surgery Hospital, Naval Medical University (Second Military Medical University), Shanghai, \\ China. \\ 3. Department of Molecular Diagnosis, Clinical Medical College, Yangzhou University, Jiangsu, China. \\ *These authors contributed equally to this work. \\ $\square$ Corresponding authors: Dr. Feng Shen, Department of Hepatic Surgery IV, the Eastern Hepatobiliary Surgery Hospital, Naval Medical University (Second \\ Military Medical University), Shanghai, China, 225 Changhai Road, Shanghai, 200433, China. Tel: 0086-21-81875005; Fax: 086-21-65562400; E-mail: \\ shenfengehbh@sina.com. Dr. Kui Wang, Department of Hepatic Surgery II, the Eastern Hepatobiliary Surgery Hospital, Naval Medical University (Second \\ Military Medical University), 225 Changhai Road, Shanghai, 200433, China. Tel: 0086-21-81875511; E-mail: wangkuiyk1@163.com.
}

(c) The author(s). This is an open access article distributed under the terms of the Creative Commons Attribution License (https://creativecommons.org/licenses/by/4.0/). See http://ivyspring.com/terms for full terms and conditions.

Received: 2020.04.02; Accepted: 2020.07.11; Published: 2020.07.25

\begin{abstract}
Background: This study aimed to establish a model predicting the prognosis of intrahepatic cholangiocarcinoma (ICC) patients with cirrhosis before liver resection (LR).

Methods: An Eastern Hepatobiliary Surgery Hospital (EHBH) model using the preoperative factors was established in a training cohort (305 patients from 2006 to 2011) and validated in an internal validation cohort (113 patients from 2012 to 2014). Predictive performance and discrimination were evaluated and compared with other staging systems.

Results: The EHBH model containing preoperative factors of carbohydrate antigen 19-9 (CA19-9), radiological tumor diameter, tumor number, and satellite nodules outperformed other staging systems in predicting the prognosis of ICC. A contour plot of 3-year survival probability and a nomogram to form two differentiated groups of patients (high-risk group and low-risk group) were constructed based on the $\mathrm{EHBH}$ model to help surgeons predicting the overall survival (OS) before LR. Patients from the high-risk group (>86.56 points) in the training cohort had worse OS rates compared with those from the low-risk group ( $\leq 86.56$ points). The one-, three-, and five-year OS rates were $50.4 \%, 29.0 \%$, and $21.0 \%$ for the high-risk group and $68.2 \%, 45.5 \%$, and $39.7 \%$ for the low-risk group, respectively $(P<0.001)$. The same results were obtained in the internal validation patients.

Conclusion: The contour plot is an easy-to-use tool to individually show the 3-year prognosis of ICC patients with different preoperative CA19-9 values and radiological characteristics before surgery. The $\mathrm{EHBH}$ model was suitable for selecting cirrhotic patients for LR to acquire a better survival.
\end{abstract}

Key words: intrahepatic cholangiocarcinoma, cirrhosis, liver resection, prognosis

\section{Introduction}

Intrahepatic cholangiocarcinoma (ICC) is a highly aggressive liver malignancy disease. The incidence of ICC, which is only second to hepatocellular carcinoma (HCC), has increased globally over the past decades [1, 2]. ICC frequently combined with large and/or multiple tumors because of late diagnoses which are characterized as negative prognostic factors [3, 4]. At present, liver resection (LR) is the only established treatment to achieve the possible long-term survival of ICC patients [5]. However, even if the patient underwent radical surgery, early postoperative recurrence of ICC usually led to a poor long-term survival outcome. 
Presently, the best efficient management for patients with multiple tumors remains controversial, especially for those with cirrhosis [6], and tumor multinodularity is attracting considerable attention because of its prognostic value in ICC [6-12]. If we could select proper patients for LR to help them acquire a good postoperative survival, some patients with poor postoperative prognosis would avoid meaningless surgery.

To that end, our study presented a simple model (Eastern Hepatobiliary Surgery Hospital model, EHBH model) based on preoperative radiological examinations and serological index, which was further validated using the internal validation cohort to accurately predict the long-term survival in cirrhotic patients before surgery. The proposed model can serve as a supplement to international staging systems in the selection of subgroups of ICC patients for surgical treatments.

\section{Materials and Methods}

\section{Study population}

A total of 305 consecutive patients who underwent LR for histopathologically confirmed ICC between January 2006 and December 2011 at EHBH was used as training cohort and 113 patients from the same center (between January 2012 and December 2014) was used as an internal validation cohort. This study was approved by the Institutional Ethics Committee of EHBH. Informed consent was obtained from all patients before surgery in this study.

\section{Inclusion and exclusion criteria}

The inclusion criteria for ICC patients were expressed as follows: (1) patients with good performance (ECOG 0-2 score) without obvious heart, lung, kidney, and other important organ diseases, (2) Child-Pugh grade A or B7 liver function without refractory ascites, (3) local tumor or multiple tumors in adjacent segments of the liver without any evidence of distant tumor metastasis. The segments or lobes can be removed or local excision can be performed with future liver remnant $\geq 50 \%$ using the measurement of preoperative computed tomography/magnetic resonance imaging (CT/MRI). (4) Mild to moderate cirrhosis, and esophagus and gastric varices with moderate to severe without bleeding tendency, namely, red color sign. No history of variceal bleeding, (5) platelet count $\geq 75 \times 10^{9} / \mathrm{L}$. The exclusion criteria were expressed as follows: patients with (1) Child-Pugh class C liver function, (2) incomplete pre- or post-operative data.

The results of the training cohort were validated in one internal validation cohort using the same inclusion/exclusion criteria.

\section{LR and definitions}

Routine preoperative laboratory and imaging examinations were conducted, as previously reported [3]. All operations were conducted for the complete removal of macroscopic tumors with adequate resection margins. The detailed surgical procedures were similar to our previously reported procedures [3]. Intraoperative ultrasound was routinely used. The range of hepatectomy was determined by the operating surgeons based on tumor stage, distribution, liver function, cirrhosis, and estimated volume of the future liver remnant. Dissection of regional lymph nodes was not routinely performed with small ICC who had no clinical evidence of nodal metastasis preoperatively or with patients of postoperatively pathological diagnosed ICC. Direct invasion of adjacent tissues and newly found intrahepatic nodules identified intraoperatively were removed whenever possible. Histopathological examination of surgical specimens was routinely conducted. The histopathological diagnosis of ICC was based on the WHO classification [7].

The presence of preoperative CSPH was based on the definition of standard surrogate criteria proposed by the Barcelona Clinic Liver Cancer (BCLC) classification, which was defined as the presence of esophagogastric varices or splenomegaly (diameter $>12 \mathrm{~cm}$ ) with a platelet count $<100 \times 10^{9} / \mathrm{L}$ [13].

Child-Pugh score was calculated as previously proposed [14]. Tumor stages or scores were categorized based on the revised liver cancer study group of Japan (LCSGJ) staging systems [15], the 8th edition of the American Joint Committee on Cancer (AJCC) [16], the Japanese Society of Hepato-BiliaryPancreatic Surgery (JSHBPS) staging system [17], the Fudan scoring system [18], and the Zhou scoring system for ICC [19].

\section{Follow-up and endpoints}

Patients were followed-up once every 2 months within the first 2 years after surgery and once every $3-$ 6 months. At each visit, liver and renal functions, alpha fetoprotein (AFP), carbohydrate antigen 19-9 (CA19-9), and carcinoembryonic antigen (CEA) were evaluated, and an abdominal ultrasound was performed. Contrast-enhanced CT scan or MRI was conducted once every 4-6 months or earlier if clinically indicated. The endpoints were overall survival (OS), which was calculated from the date of surgery to the date of patient death or last follow-up, and recurrence-free survival (RFS) was defined from the date of surgery to the date when HCC recurrence was first diagnosed. 


\section{Statistical analysis}

Continuous variables were reported as means with standard deviations or medians with interquartile range. They were compared using student's $t$-test or Mann-Whitney $U$ test. Categorical data, presented as frequencies (\%), were compared using chi-square or Fisher's exact tests. To establish the EHBH model and the nomogram, we selected the outcomes of multivariable Cox regression analyses of OS with a stepwise method by using the "rms" package of $\mathrm{R}$, version 3.4 (http://www.r-project. org/). Then, a restricted cubic spline (RCS) was used to find the nonlinear relationship between the variables and OS. Moreover, predicted probability and risk contour plots were used to display the predicted risk of individual patients.

We used several methods to validate the effectiveness of the EHBH model. First, the prediction accuracies of the EHBH model and other clinical other staging systems were calculated based on Harrell's c-statistics [20]. Then, the nomogram scores of each patient were calculated and then the best cut-off score of the nomogram was detected by the receiver operating characteristic curve (ROC) to divide patients into the high-risk group and low-risk group, and Kaplan-Meier curves of OS or RFS were calculated in different cohorts and groups. Decision curve analysis (DCA) of the EHBH model was made to validate its performance in the training cohort. Time-dependent areas under the receiver operating characteristic curve (AUC) of each point in different cohorts were measured from 10 months to 80 months, reflecting the performance in predicting OS at various time points. All the reported $\mathrm{p}$ values were the results of two-sided tests. A significance level of 0.05 was applied. All statistical analyses were carried out in the R program, version 3.4 (http://www.r-project.org/) (Vienna, Austria, version 3.4.4), and SPSS (IBM, version 23, USA).

\section{Results}

\section{Patient characteristics}

As shown in Figure 1, 305 patients with cirrhosis at $\mathrm{EHBH}$ met the inclusion criteria, which were enrolled in the training cohort. The internal validation cohort of 113 ICC patients was obtained from an independent cohort of consecutive patients with cirrhosis in EHBH. Table 1 summarizes the baseline clinicopathological features of the patients between the two cohorts. Significant differences were only observed in the percentage of satellite nodules among the two cohorts. Kaplan-curves of OS and RFS between the two cohorts were shown in Figures S1A, S1B.

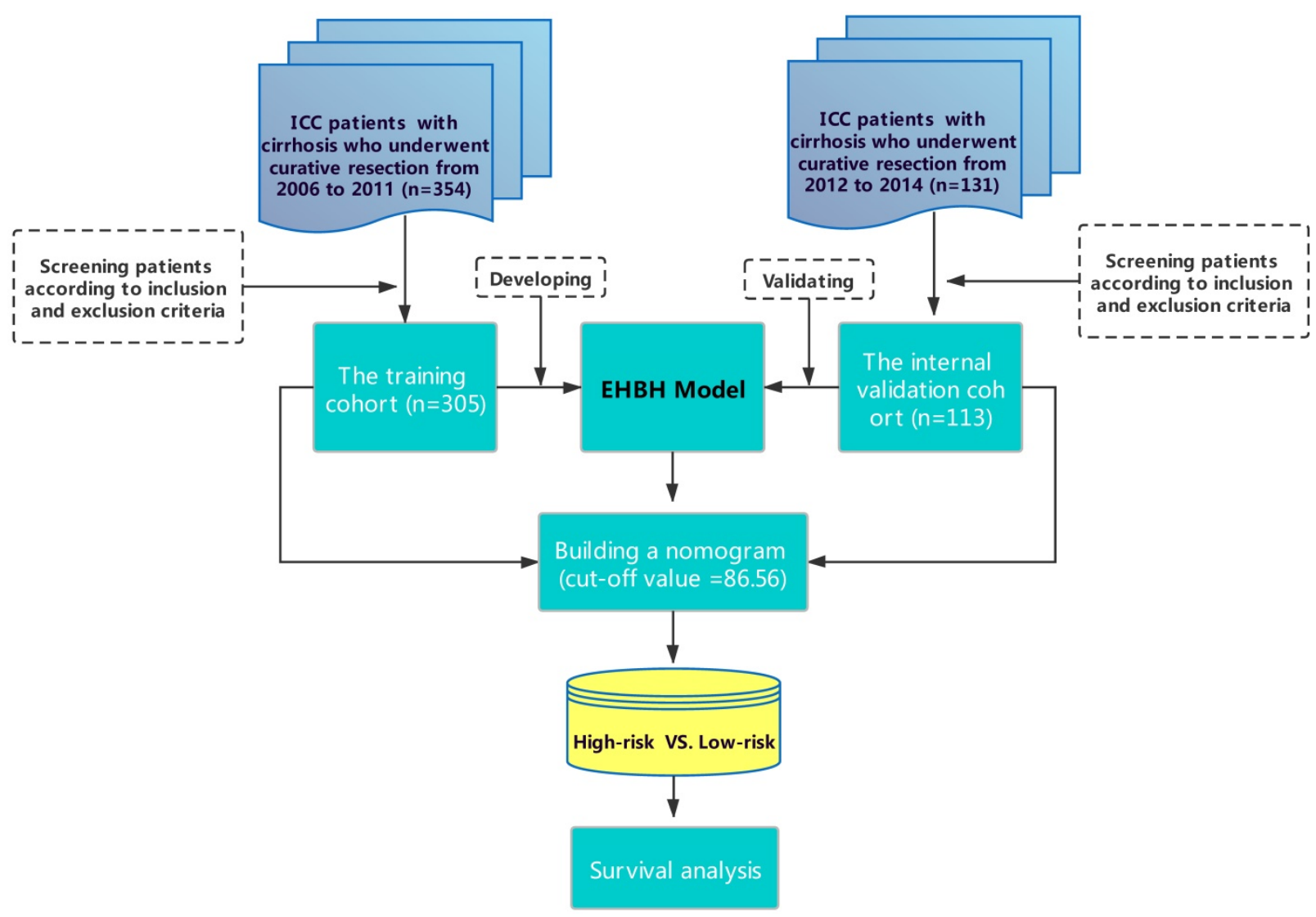

Figure 1. Flowchart of this study. 
Table 1. Baseline characteristics of the study cohorts

\begin{tabular}{|c|c|c|c|c|}
\hline Variable & Entire cohort $(n=418)$ & Training cohort $(\mathrm{n}=305)$ & Validation cohort $(n=113)$ & $P$ value \\
\hline \multicolumn{5}{|l|}{ Basical results } \\
\hline Age, Year & $51.8 \pm 10.3$ & $52.1 \pm 10.2$ & $51.2 \pm 10.5$ & 0.455 \\
\hline Gender, n (\%) & & & & 0.494 \\
\hline Male & $368.0(88.0)$ & $266.0(87.2)$ & $102.0(90.3)$ & \\
\hline Female & $50.0(12.0)$ & $39.0(12.8)$ & $11.0(9.7)$ & \\
\hline BMI & $24.2 \pm 4.0$ & $24.2 \pm 3.9$ & $24.1 \pm 4.0$ & 0.814 \\
\hline CSPH, n (\%) & & & & 0.464 \\
\hline Yes & $131.0(31.3)$ & $92.0(30.2)$ & $39.0(34.5)$ & \\
\hline No & $287.0(68.7)$ & $213.0(69.8)$ & $74.0(65.5)$ & \\
\hline Diabetes, n (\%) & & & & 0.775 \\
\hline Yes & $29.0(6.9)$ & $20.0(6.6)$ & $9.0(8.0)$ & \\
\hline No & $389.0(93.1)$ & $285.0(93.4)$ & $104.0(92.0)$ & \\
\hline HBsAg, n (\%) & & & & 0.545 \\
\hline Positive & $365.0(87.3)$ & $264.0(86.6)$ & $101.0(89.4)$ & \\
\hline Negative & $53.0(12.7)$ & $41.0(13.4)$ & $12.0(10.6)$ & \\
\hline HCV-Ab, n (\%) & & & & 1.000 \\
\hline Positive & $10.0(2.4)$ & $7.0(2.3)$ & $3.0(2.7)$ & \\
\hline Negative & $408.0(97.6)$ & $298.0(97.7)$ & $110.0(97.3)$ & \\
\hline \multicolumn{5}{|l|}{ Serological results } \\
\hline $\mathrm{TBIL}, \mathrm{mg} / \mathrm{dL}^{*}$ & $0.8(0.6,1.0)$ & $0.8(0.6,1.0)$ & $0.7(0.7,1.1)$ & 0.366 \\
\hline $\mathrm{ALB}, \mathrm{g} / \mathrm{L}$ & $41.3 \pm 4.9$ & $41.2 \pm 4.8$ & $41.5 \pm 5.0$ & 0.574 \\
\hline $\mathrm{ALT}, \mathrm{IU} / \mathrm{L}^{*}$ & $35.5(25.6,56.0)$ & $35.3(25.5,55.6)$ & $36.4(25.7,58.0)$ & 0.636 \\
\hline AST, IU $/ L^{*}$ & $32.1(25.0,45.6)$ & $31.9(25.0,44.2)$ & $32.7(25.0,51.1)$ & 0.261 \\
\hline GGT, IU/L* & $67.5(40.0,149.0)$ & $67.0(41.0,148.5)$ & $75.0(38.0,151.0)$ & 0.922 \\
\hline PT, second * & $12.2(11.7,13.0)$ & $12.2(11.7,13.0)$ & $12.2(11.7,13.1)$ & 0.642 \\
\hline PLT, $\times 10^{9} / \mathrm{L}$ & $151.45 \pm 64.39$ & $152.54 \pm 65.28$ & $148.51 \pm 62.13$ & 0.571 \\
\hline $\mathrm{AFP}, \mu \mathrm{g} / \mathrm{L}^{*}$ & $8.6(3.7,65.2)$ & $8.4(3.6,56.1)$ & $9.8(4.0,112.5)$ & 0.296 \\
\hline $\mathrm{CEA}, \mu \mathrm{g} / \mathrm{L}^{*}$ & $2.6(1.7,4.2)$ & $2.5(1.6,4.1)$ & $2.7(1.7,4.7)$ & 0.452 \\
\hline CA19-9, IU/mL* & $39.5(17.9,121.0)$ & $39.7(18.1,124.3)$ & $36.4(17.3,92.7)$ & 0.503 \\
\hline Child-Pugh grade, $\mathbf{n}(\%)$ & & & & 0.781 \\
\hline A & $384.0(91.9)$ & $279.0(91.5)$ & $105.0(92.9)$ & \\
\hline B & $34.0(8.1)$ & $26.0(8.5)$ & $8.0(7.1)$ & \\
\hline \multicolumn{5}{|l|}{ Imaging results } \\
\hline Tumor diameter, $\mathrm{cm}^{*} \dagger$ & $5.0(3.5,8.0)$ & $5.0(3.6,8.0)$ & $4.9(3.2,8.5)$ & 0.995 \\
\hline Tumor distribution, $\mathbf{n}(\%)$ & & & & 0.887 \\
\hline Bilateral & $59.0(14.1)$ & $44.0(14.4)$ & $15.0(13.3)$ & \\
\hline Unilateral & $359.0(85.9)$ & $261.0(85.6)$ & $98.0(86.7)$ & \\
\hline Number of tumor nodal & & & & 0.967 \\
\hline Multiple & $169.0(40.4)$ & $124.0(40.7)$ & $45.0(39.8)$ & \\
\hline Sigle & $249.0(59.6)$ & $181.0(59.3)$ & $68.0(60.2)$ & \\
\hline Satellite nodules, $\mathrm{n}(\%)$ & & & & 0.048 \\
\hline Yes & $96.0(23.0)$ & $62.0(20.3)$ & $34.0(30.1)$ & \\
\hline No & $322(77.0)$ & $243(79.7)$ & $79(69.9)$ & \\
\hline Tumor capsule, $n(\%)$ & & & & 0.718 \\
\hline No & $342(81.8)$ & $252(82.6)$ & $90(79.6)$ & \\
\hline Complete & $37(8.9)$ & $25(8.2)$ & $12(10.6)$ & \\
\hline Incomplete & $39(9.3)$ & $28(9.2)$ & $11(9.7)$ & \\
\hline
\end{tabular}

\section{Univariable and multivariable Cox analyses}

For the univariable analysis, presence of CSPH $(\mathrm{HR}=1.399, \quad 95 \% \mathrm{CI}=1.038-1.886, \quad p=0.027), \quad$ high CA19-9 value $(\mathrm{HR}=1.001 ， 95 \% \mathrm{CI}=1.000-1.002$, $p<0.001)$, large tumor diameter $(\mathrm{HR}=1.118$, 95\% CI $=1.078-1.159, p<0.001)$, more tumor numbers $(\mathrm{HR}=1.437, \quad 95 \% \mathrm{CI}=1.287-1.604, \quad p<0.001), \quad$ and presence of satellite nodules $(\mathrm{HR}=1.747$, $95 \% \mathrm{CI}=1.257-2.429, p=0.001)$ were related to OS in the training cohort (Table 2). After multivariable Cox regression analysis, high CA19-9 value $(\mathrm{HR}=1.001$, $95 \% \mathrm{CI}=1.000-1.002, p<0.001)$, large tumor diameter $(\mathrm{HR}=1.088, \quad 95 \% \mathrm{CI}=1.046-1.132, \quad p<0.001), \quad$ more tumor numbers $\quad(\mathrm{HR}=1.323, \quad 95 \% \mathrm{CI}=1.174-1.492$, $p<0.001)$, and satellite nodules (HR=1.759, $95 \% \mathrm{CI}=1.255-2.466, p=0.001)$ were the independent risk factors of OS (Table 2 and Figure 2A). As shown in Figure 2B, RCS showed that nonlinear relationships existed between the factors of CA19-9, radiological tumor diameter, and tumor number and OS rate in ICC patients with cirrhosis. As the tumor diameter and number increased, the patient's risk of death increased. However, when CA19-9 > $500 \mathrm{IU} / \mathrm{mL}$, the risk of death decreased slightly. In addition, the results of RFS in univariable and multivariable Cox analyses are shown in Table S1. 


\section{Establishment of the EHBH model}

We presented a simple EHBH model consisted of a serological index and three preoperative imaging features, namely, CA19-9, tumor diameter, tumor number, and satellite nodules, based on the result of multivariable analysis for OS in the training cohort. The EHBH model can be used to visually predict the overall 3-year survival probabilities of all patients with cirrhosis, as shown in Figures $3 \mathrm{~A}$ and $3 \mathrm{~B}$ with two contour plots. The contour plot included two steps. First, the specific contour plot should be selected for the patients whether with or without satellite nodules (without satellite nodules $=\mathrm{A}$, with satellite nodules $=\mathrm{B}$ ). Second, the predicted probability was traced by tumor number + tumor diameter (X-axis) and CA19-9 value (Y-axis) in the specific contour plot. Moreover, the one- and five-year predicted survival probabilities are shown in
Figure S2, which used the same way as previously described.

Based on the EHBH model, a nomogram was built for individual patient survival risk stratification (Figure 4). The 1-year, 3-year, and 5-year survival probabilities of individual patients could be predicted before the surgery with the sum of CA19-9 value, tumor size, tumor number, and satellite nodules.

\section{Risk stratification and selection for optimal surgical patients}

To indicate the efficacy of the $\mathrm{EHBH}$ model to select reasonably ICC patients with cirrhosis for hepatectomy, we calculated the nomogram score for each patient in the training cohort and through ROC curves found the best cut-off value of 86.56 points, and then divided the patients into high-risk and low-risk groups, as shown in Figure S3.

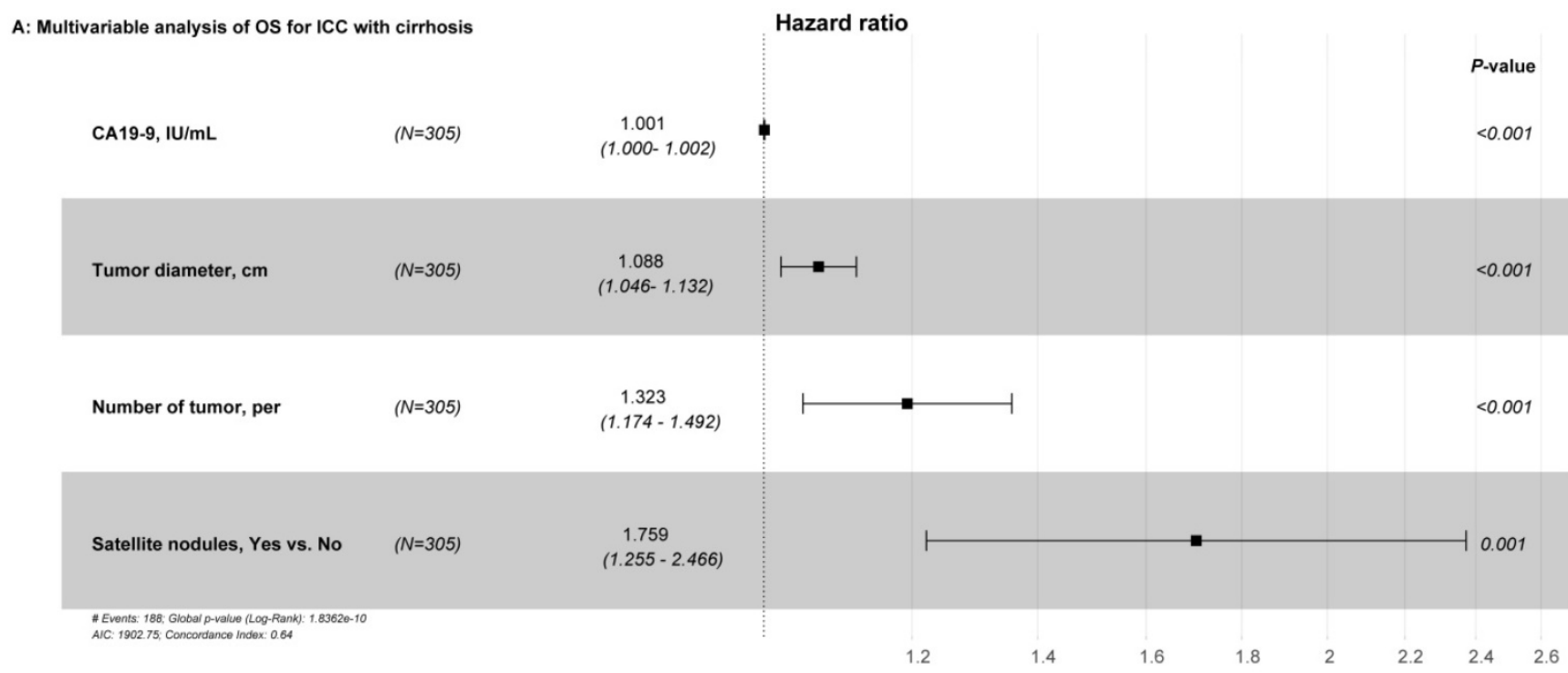

B: Restricted cubic splines of CA19-9, tumor diameter and number of tumor
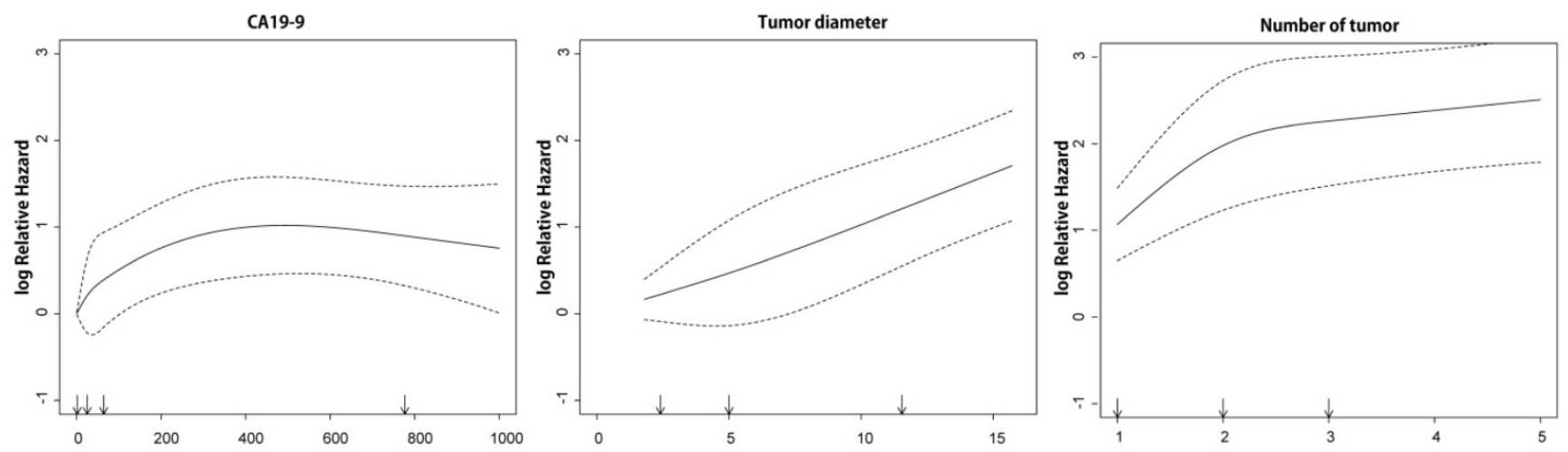

Figure 2. Relationships between the variables and OS rate of patients with ICC and cirrhosis. A: Forest plots summarizing the multivariable result of OS; B: Nonlinear relationships of CA19-9 value, tumor diameter, and number with OS of patients with ICC and cirrhosis. 
Table 2. Univariable and multivariable analyses of overall survival

\begin{tabular}{|c|c|c|c|c|c|c|}
\hline \multirow[t]{2}{*}{ Variable } & \multicolumn{3}{|c|}{ Univariable analysis } & \multicolumn{3}{|c|}{ Multivariable analysis } \\
\hline & HR & $95 \% \mathrm{CI}$ & $P$ value & HR & $95 \% \mathrm{CI}$ & $P$ value \\
\hline Age, year & 1.001 & $0.988-1.015$ & 0.846 & & & \\
\hline Gender, Male vs. Female & 1.327 & $0.842-2.090$ & 0.221 & & & \\
\hline BMI & 0.977 & $0.942-1.014$ & 0.223 & & & \\
\hline CSPH, Yes vs. No & 1.399 & $1.038-1.886$ & 0.027 & & & \\
\hline Diabetes, Yes vs. No & 1.363 & $0.790-2.352$ & 0.263 & & & \\
\hline HBsAg, Yes vs. No & 0.966 & $0.643-1.453$ & 0.870 & & & \\
\hline Anti-HCV, Yes vs. No & 0.517 & $0.165-1.619$ & 0.249 & & & \\
\hline TBIL, mg/dL & 1.019 & $0.964-1.076$ & 0.504 & & & \\
\hline $\mathrm{ALB}, \mathrm{g} / \mathrm{L}$ & 0.987 & $0.958-1.018$ & 0.423 & & & \\
\hline ALT, IU/L & 1.000 & $0.999-1.001$ & 0.416 & & & \\
\hline AST, IU/L & 1.000 & $0.999-1.001$ & 0.263 & & & \\
\hline GGT, IU/L & 1.000 & $0.999-1.001$ & 0.920 & & & \\
\hline PT, second & 1.071 & $0.953-1.203$ & 0.250 & & & \\
\hline PLT,$\times 10^{9} / \mathrm{L}$ & 1.000 & $0.998-1.003$ & 0.729 & & & \\
\hline $\mathrm{AFP}, \mu \mathrm{g} / \mathrm{L}$ & 1.000 & $0.999-1.001$ & 0.759 & & & \\
\hline $\mathrm{CEA}, \mu \mathrm{g} / \mathrm{L}$ & 1.001 & $0.999-1.003$ & 0.101 & & & \\
\hline CA19-9, IU/mL & 1.001 & $1.000-1.002$ & $<0.001$ & 1.001 & $1.000-1.002$ & $<0.001$ \\
\hline Child-Pugh grade, B vs. A & 1.164 & $0.716-1.893$ & 0.539 & & & \\
\hline Tumor diameter, $\mathrm{cm}$ & 1.118 & $1.078-1.159$ & $<0.001$ & 1.088 & 1.046-1.132 & $<0.001$ \\
\hline Tumor distribution, Bilateral vs. Unilateral & 1.212 & $0.825-1.783$ & 0.326 & & & \\
\hline No. of tumor, per & 1.437 & $1.287-1.604$ & $<0.001$ & 1.323 & $1.174-1.492$ & $<0.001$ \\
\hline Satellite nodules, Yes vs. No & 1.747 & $1.257-2.429$ & 0.001 & 1.759 & $1.255-2.466$ & 0.001 \\
\hline Tumor capsule, Yes vs. No & 0.843 & $0.652-1.089$ & 0.189 & & & \\
\hline
\end{tabular}

As shown in Figure 5A, the patients' survival outcomes of the high-risk group $(n=119)$ were significantly worse than those of the low-risk group $(\mathrm{n}=186)$ (median time, 11.91 vs. 28.39 months, $\mathrm{p}<0.001$ ) in the training cohort. The one-, three-, and five-year OS rates were $50.4 \%, 29.0 \%$, and $21.0 \%$ for the high-risk group and $68.2 \%, 45.5 \%$, and $39.7 \%$ for the low-risk group, respectively. The one-, three-, and five-year RFS rates using the same cut-off value of 86.56 in the training cohorts were $38.9 \%, 28.7 \%$, and $20.2 \%$ for the high-risk group and $55.6 .9 \%, 35.2 \%$, and $32.5 \%$ for the low-risk group, respectively $(p=0.004)$, as shown in Figure S1C.

\section{Assessment and comparison of the EHBH model}

Several methods were used to verify the predictive accuracy of the EHBH model. First, DCA was used to facilitate the comparison between the EHBH model and other staging systems (Child-Pugh stage, LCSGJ stage, AJCC 8th stage, JSHBPS stage, Fudan score, and Zhou score) in the training cohort. As shown in Figure 5B, DCA demonstrated that the EHBH-HVTT scoring system provided superior net benefit when the threshold value $>0.2$ compared with other commonly used international staging systems. Table S2 shows that the EHBH model with the highest C-index (C-index $=0.710,95 \% \mathrm{CI}=0.673-0.748$ ) was significantly better than the common staging systems under Harrell's c-statistics (all p < 0.0001). Furthermore, time-dependent-ROC curve area analysis was used to determine which staging systems were good at predicting the survival outcomes. As shown in Figure 5C, the EHBH model outperformed the other six currently available models in the training cohort. The time-dependent AUC ranging from 10 months to 80 months for the EHBH model was 0.68 (0.59-0.78) in the training cohort.

These results demonstrated that the $\mathrm{EHBH}$ model had a higher diagnostic capacity and better AUC than the other six staging systems in cirrhotic patients with ICC. The predicted plots of OS probabilities (one-, three-, and five-year) in each clinical staging system for all cirrhotic ICC patients are shown in Figure 5D and Figure S4. The EHBH model showed a significant relationship with the largest Wald and Slope with the OS rate of patients compared with other staging systems.

\section{Validation of the EHBH model}

Using the same threshold of 86.56 points, the identification ability of the internal validation cohort performed equally well with the $\mathrm{EHBH}$ model established in the training cohort. In the internal validation cohort, ICC patients in the low-risk group $(n=69)$ had a longer median OS than that in the high-risk group $(\mathrm{n}=44)$ (Figure 6A, median time, 41.05 vs. 10.47 months, $\mathrm{p}<0.001$ ). The EHBH model can easily distinguish the optimal patients with better RFS $(p=0.040) \quad$ (Figure S1D). Moreover, timedependent AUC ranging from 10 months to 80 months of the EHBH model in the internal validation cohort was $0.72(0.64-0.82)$ (Figure 6B). As shown in Figure 6B, the AUC value of the $\mathrm{EHBH}$ 
model was the highest in all staging systems at a time range from 10 months to 73 months.

\section{Discussion}

Cirrhosis is regarded as an important negative prognostic factor for the survival of ICC patients. The incidence of cirrhosis has been estimated to be $27.8 \%$ to $50.5 \%$ in ICC patients [21, 22]. With the advancement of surgical techniques [22-27], more and more cirrhotic patients are suitable for surgery. However, survival outcomes are still controversial, especially in patients with multiple tumors [28].

\section{A: 3-year overall survival rate of cirrhotic ICC without satellite nodules}

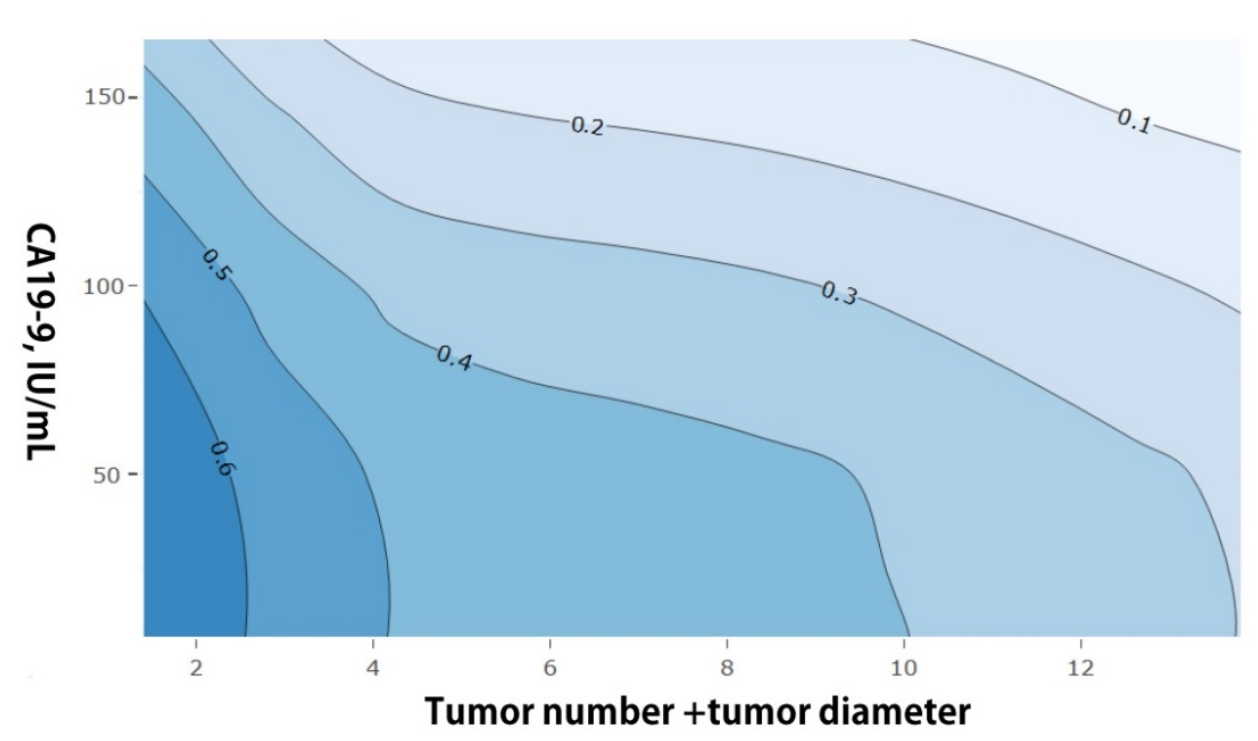

Survival probability

\section{B: 3-year overall survival rate of cirrhotic ICC with satellite nodules}

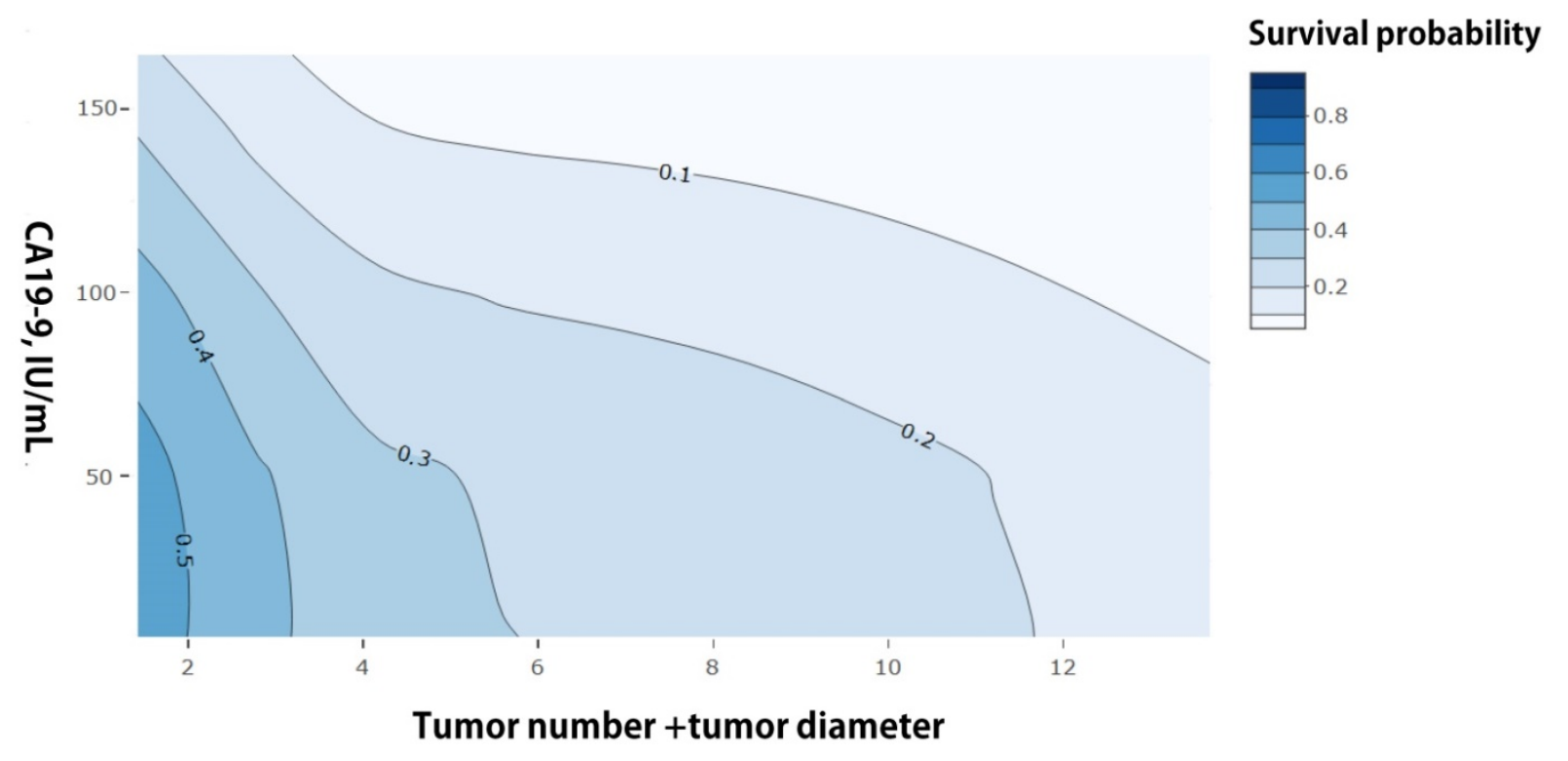

Figure 3. Contour plots of three-year using the EHBH model for ICC patients with cirrhosis. A: For patients without satellite nodules. B: For patients with satellite nodules. 
Points

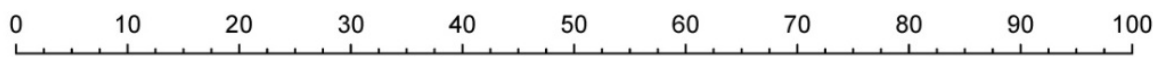

CA19-9, IU/mL

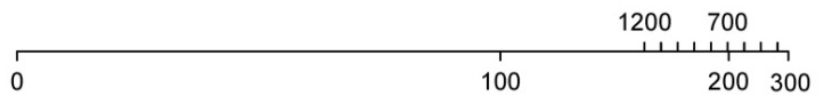

Tumor diameter, $\mathbf{c m}$

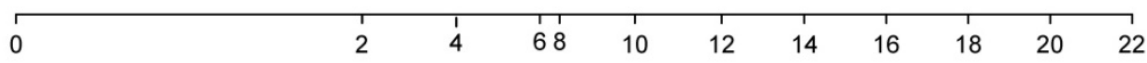

Number of tumor, pre

Satellite nodules

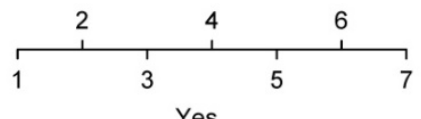

Yes

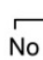

Total Points

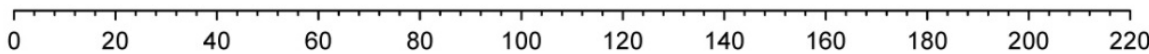

1-year survival Probability

$\begin{array}{llllllllll}0.9 & 0.8 & 0.7 & 0.6 & 0.5 & 0.4 & 0.3 & 0.2 & 0.1\end{array}$

3-year survival Probability

$\begin{array}{lllllllll}0.8 & 0.7 & 0.6 & 0.5 & 0.4 & 0.3 & 0.2 & 0.1\end{array}$

5-year survival Probability

$\begin{array}{llllllllll}0.8 & 0.7 & 0.6 & 0.5 & 0.4 & 0.3 & 0.2 & 0.1\end{array}$

Figure 4. Nomogram of the EHBH model for individual survival prediction.

To the best of our knowledge, this study was the first to establish a simple model using preoperative indexes for the decision making on hepatectomy and prognosis prediction of ICC patients with cirrhosis, especially for those with multiple tumors. A few of previous studies have established preoperative prognosis models for ICC, however, some studies just use single preoperative indicators or indexes to predict the prognosis of ICC, such as albuminbilirubin grade (ALBI), albumin-to-alkaline phosphatase ratio (AAPR) [29], and preoperative prognostic nutritional index (PNI) [30]. Those indexes did not contain any information about tumor characteristics, such as tumor size, number, and so on. Some studies use multivariable regression to select significant factors and then develop a risk score to accomplish survival prediction [31, 32]. Compared with ours, those risk score models require complex calculation process in clinical application. In the contrary, our EHBH model just consists of three factors, and the contour plots are easily used to estimate the survival probability of ICC patients. What' more, a nomogram can be used to distinguish the high-risk cirrhotic patients before surgery. The patients in the low-risk group (nomogram score $\leq$ 86.56 points) were good candidates for LR because they would have good median OS and RFS. A contour plot was used as a visualization tool for surgeons and patients to accomplish individualized prognosis prediction of 3-year survival probability based on preoperative CA19-9 value, tumor number + tumor diameter, and satellite nodules. Most importantly, the $\mathrm{EHBH}$ model performed better in the prognosis prediction than the other commonly used staging systems.

Cumulative evidences have proved CA19-9 useful to help surgeons selecting proper patients with intrahepatic cholangiocarcinoma (ICC) for surgery and an important preoperative biomarker for prognostic prediction after surgical treatment. For example, Yamamoto et al. [33] suggested that therapy or resection should be carefully determined in ICC patients with CA19-9 > $300 \mathrm{U} / \mathrm{ml}$. Moreover, a study from $\mathrm{He}$ et al. [34] showed that ICC patients with preoperative CA19-9 value $>200 \mathrm{U} / \mathrm{ml}$ generally had a poor surgical result. In this study, our conclusion was consistent with the previous. A higher level of preoperative CA19-9 value was significantly associated with worse prognosis after LR in cirrhotic ICC patients.

Tumor size and number were also considered as important risk factors of ICC based on Wang's nomogram [3]. Recently, a multicenter study demonstrated the linear effect of tumor size on 
survival [8]. These findings supported the wellestablished relationship between tumor size and prognosis of HCC [35]. Controversies have existed about the relationship between tumor number and survival in ICC after surgery. Some authors have identified multiple tumors as a negative prognostic factor [6-11]. For example, Pietro et al. [36] indicated that multiple tumors are common in ICC patients who are characterized by a decrease in OS and DFS and a trend toward early recurrence. Other researchers have not considered that tumor number is a risk factor for survival [12].

\section{A: K-M curves of OS in training cohort}

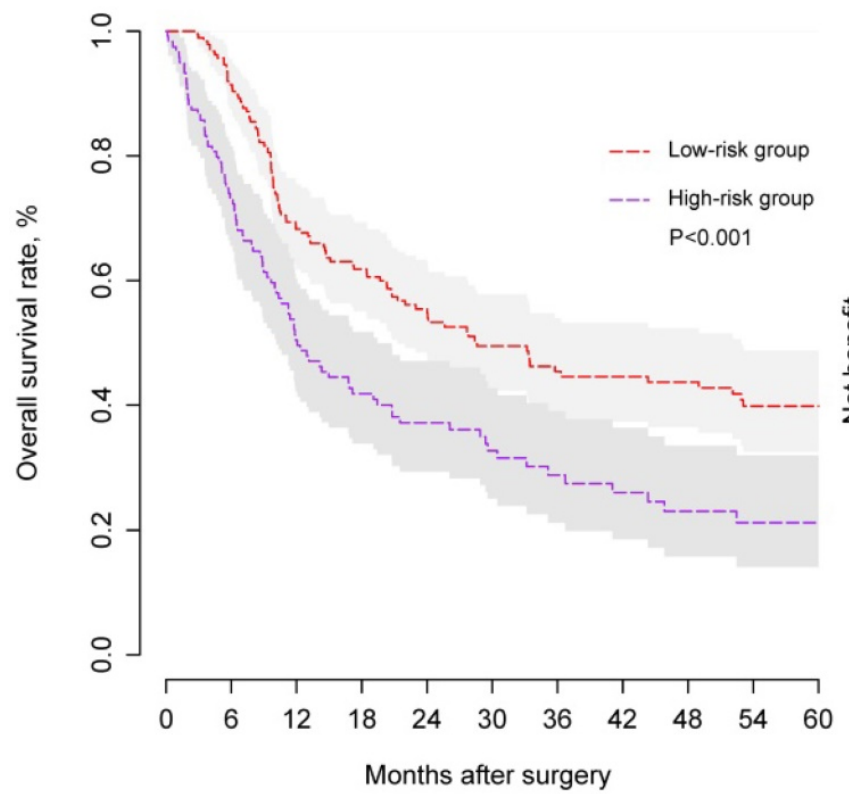

C:Time-dependent AUROC values of models in training cohort

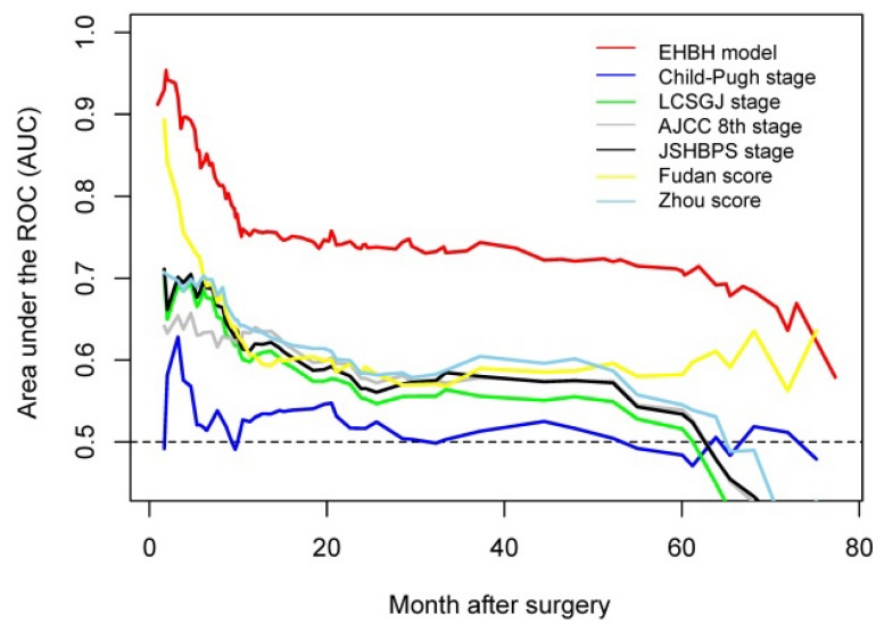

Some researchers have pointed out that tumor multinodularity is associated with other poor prognostic factors in ICC [37]. Pietro et al. [36] also observed that tumor multinodularity is associated with the increase in LNM rate, perineural, and vascular invasion, leading to poor prognosis of patients after LR. What's more, tumor multinodularity was associated with the highest rate of LNMs $(62 \%)$, confirming similar findings of two previous studies $[9,12]$. In this study, we observed that tumor diameter and tumor number reflected the poor prognosis of ICC on multivariable analyses.

\section{B: Decision curve analysis}

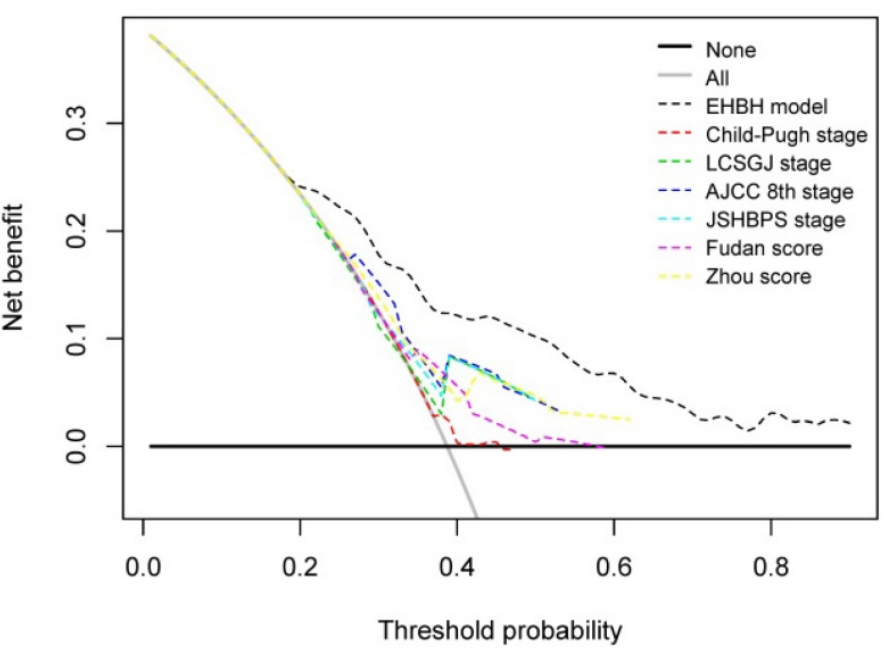

D: Predicted probability of death

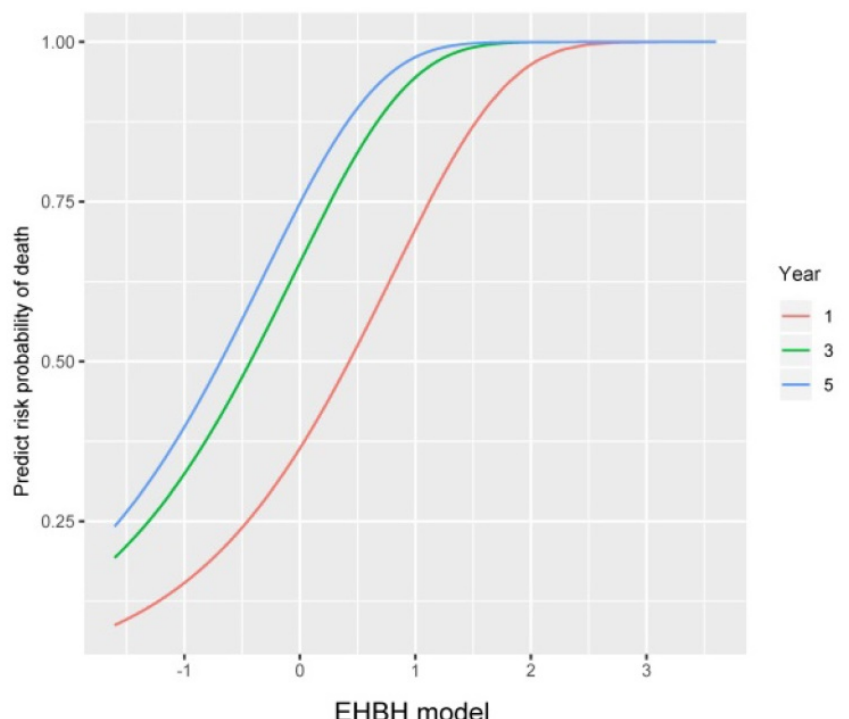

Figure 5. Establishment and assessment of the EHBH model in the training cohort. A: Kaplan-Meier curve of the stratified EHBH model in the training cohort. B: DCA of the EHBH model. C: Time-dependent ROC for the EHBH model and other clinical staging systems in the training cohort. D: Predicted probability of one-, three-, and five-year death using the $\mathrm{EHBH}$ model. 
A: K-M curves of OS in internal validation cohort

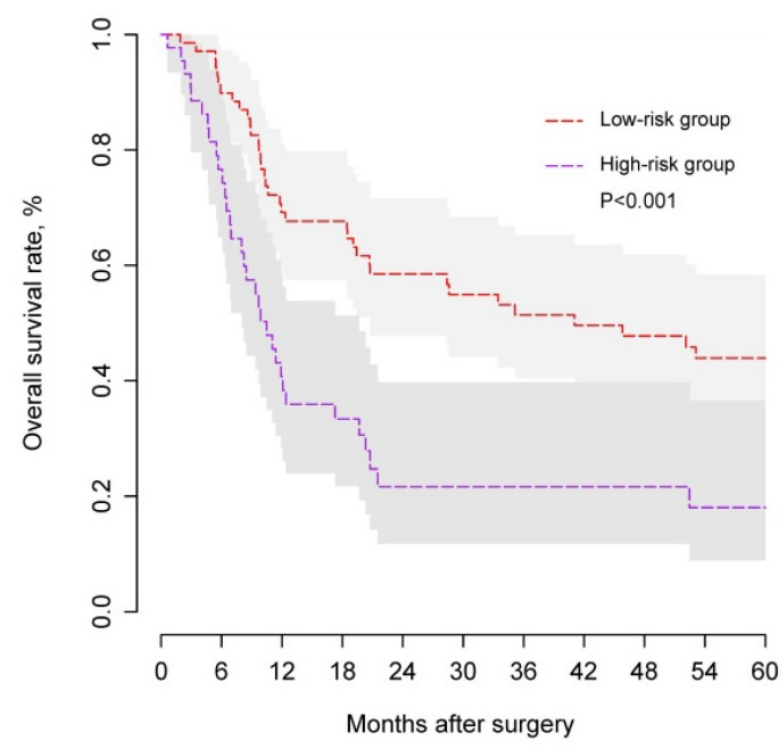

B: Time-dependent AUROC values of models in internal validation cohort

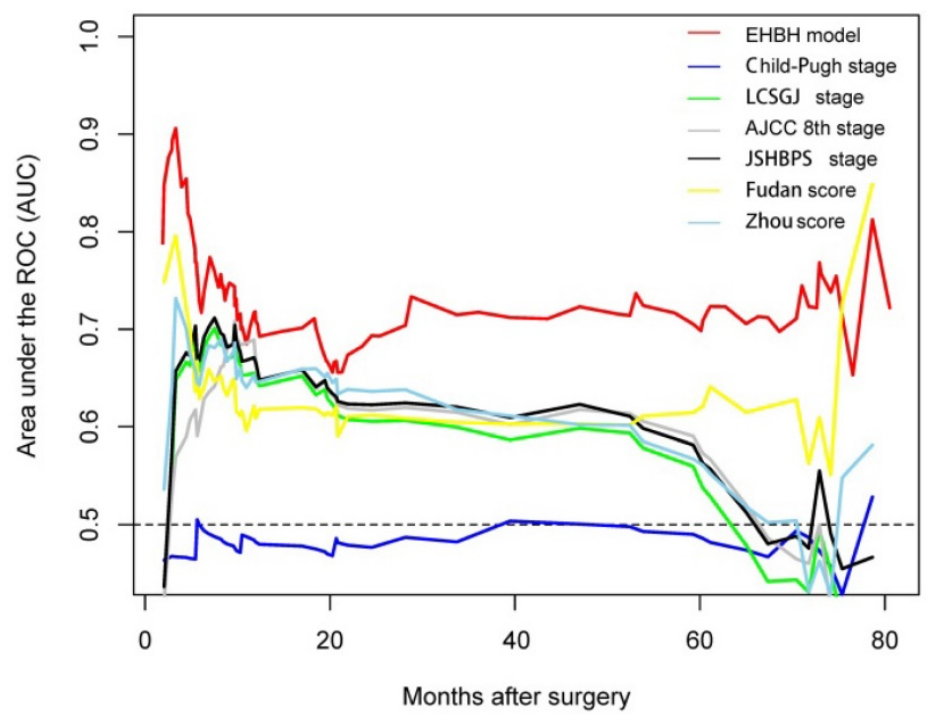

Figure 6. Assessment of the EHBH model in the internal validation cohort. A: Kaplan-Meier curve of the stratified EHBH model in the internal validation cohort. B: Time-dependent ROC for the EHBH model and other clinical staging systems in the internal validation cohort.

Recently, many studies have shown that satellite nodules are an important risk predictor of survival in patients with ICC [36, 38-40]. Our results showed that satellite nodules could increase the death rate in ICC patients which are consistent with the recent study by $\mathrm{Lu}$ et al. [38]. The research of Zhang et al. [40] also suggested that satellite lesions were an independent risk factor for recurrence in ICC.

Satellite nodules might be a presence of vascular infiltration suggesting a more aggressive form in ICCs, which could explain why patients with satellite nodules received worse survival outcomes in our study. Besides, satellite nodules usually combined with a higher rate of multiple tumors in ICC patients $[36,38]$. In the present study, we also found that the percentage of patients having $>2$ nodules with satellite nodules was more than that without satellite nodules.

This study compared six common international staging systems to stratify ICC patients into different risk categories using the $\mathrm{EHBH}$ model. We found that those six staging systems had poor prediction capability of OS. This condition was because some systems were originally developed from mixed populations, and only part of patients involved in the establishment of those systems was suitable for surgery. On the other hand, the establishment of those systems just based on the clinicopathological data from hundreds of ICC patients.

The Child-Pugh system was originally developed to assess the prognosis of patients with cirrhosis and portal hypertension who were assessed to undergo surgery for variceal bleeding. Some of the variables included in this system were interrelated (e.g., ascites and serum albumin levels). Furthermore, the grading of ascites and encephalopathy was subjective [41].

The other five clinical staging systems, namely, LCSGJ stage, AJCC 8th stage, JSHBPS stage, Fudan score, and Zhou score based on tumor characteristics and blood biochemical had some limitations such as lacking external validations, including patients who were unsuitable for surgery and so on. Besides, these staging systems had limitations because they cannot be used to make an individualized prediction of survival probability before LR for ICC patients with cirrhosis. Our EHBH model can solve those problems using the contour plot of predicted 3-year survival probability based on the result of multivariable Cox regression in ICC before surgery. This model can identify cirrhotic ICC patients with proper tumor number and size suitable for surgery.

The EHBH model has four significant advantages in clinical practice compared with other staging systems for ICC patients with cirrhosis. First, the model is simple and only contains four variables. Second, the EHBH model aids surgeons to select suitable patients for LR. Third, the contour plot can achieve individualized prediction for long-term survival. Lastly, this new system is stable and accurate in predicting the OS of cirrhotic ICC patients. Taken together, the EHBH model can be used as a supplement to the commonly used international staging systems to improve their prognostic ability in cirrhotic ICC patients. 
The current study had several limitations. First, it only involved one single institution in China, and patients were all cirrhotic background. The model should be validated using more external cohorts with different backgrounds. Second, the EHBH model using preoperative radiological indexes need to further improve the accuracy of prognosis prediction with more preoperative parameters. Third, this retrospective study was inevitably exposed to selection bias, and other potential confounding factors that may have prognostic roles were not adjusted.

All in all, the EHBH model was used as a selection aid for surgeons to screen cirrhotic ICC patients suitable for hepatectomy and predict the individual survival probability of ICC patients with cirrhosis, which can be used as a supplement to other ICC staging systems.

\section{Supplementary Material}

Supplementary figures and tables. http://www.jcancer.org/v11p5623s1.pdf

\section{Acknowledgements}

\section{Funding}

This study was supported by grants from the State Key Project on Infectious Diseases of China (2018ZX10723204-001); Joint Tackling Project of Emerging Frontier Technologies in Shanghai Hospitals in 2017 (SHDC12017122).

\section{Author Contributions}

Feng Shen and Kui Wang are responsible for the integrity of the data and the accuracy of the data analyses. Qinjunjie Chen, Fengwei Li, and Yuzhen Gao: study concept and design, and drafting of the manuscript; Hui Xue and Zheng Li: collecting and analysis of data; Qifei Zou and Yong Xia: providing study material or patients; Feng Shen and Kui Wang: critical revision of the manuscript for important intellectual content and administrative support. All authors have read and approved the final version of the manuscript.

\section{Compliance with ethical standards}

The study was conducted ethically under the World Medical Association Declaration of Helsinki and was approved by the Institutional Ethics Committee of EHBH.

\section{Competing Interests}

The authors have declared that no competing interest exists.

\section{References}

1. Siegel RL, Miller KD, Jemal A. Cancer Statistics, 2017. CA Cancer J Clin. 2017; 67: 7-30.

2. Mavros MN, Economopoulos KP, Alexiou VG, Pawlik TM. Treatment and Prognosis for Patients With Intrahepatic Cholangiocarcinoma: Systematic Review and Meta-analysis. JAMA Surg. 2014; 149: 565-74.

3. Wang Y, Li J, Xia Y, Gong R, Wang K, Yan Z, et al. Prognostic nomogram for intrahepatic cholangiocarcinoma after partial hepatectomy. J Clin Oncol. 2013; 31: 1188-95.

4. Bridgewater J, Galle PR, Khan SA, Llovet JM, Park JW, Patel T, et al. Guidelines for the diagnosis and management of intrahepatic cholangiocarcinoma. J Hepatol. 2014; 60: 1268-89.

5. Amini N, Ejaz A, Spolverato G, Kim Y, Herman JM, Pawlik TM. Temporal trends in liver-directed therapy of patients with intrahepatic cholangiocarcinoma in the United States: a population-based analysis. J Surg Oncol. 2014; 110: 163-70.

6. Wright GP, Perkins S, Jones H, Zureikat AH, Marsh JW, Holtzman MP, et al. Surgical Resection Does Not Improve Survival in Multifocal Intrahepatic Cholangiocarcinoma: A Comparison of Surgical Resection with Intra-Arterial Therapies. Ann Surg Oncol. 2018; 25: 83-90.

7. de Jong MC, Nathan H, Sotiropoulos GC, Paul A, Alexandrescu S, Marques H, et al. Intrahepatic cholangiocarcinoma: an international multi-institutional analysis of prognostic factors and lymph node assessment. J Clin Oncol. 2011; 29: 3140-5.

8. Hyder O, Marques H, Pulitano C, Marsh JW, Alexandrescu S, Bauer TW, et al. A nomogram to predict long-term survival after resection for intrahepatic cholangiocarcinoma: an Eastern and Western experience. JAMA Surg. 2014; 149: 432-8.

9. Ribero D, Pinna AD, Guglielmi A, Ponti A, Nuzzo G, Giulini SM, et al. Surgical Approach for Long-term Survival of Patients With Intrahepatic Cholangiocarcinoma: A Multi-institutional Analysis of 434 Patients. Arch Surg. 2012; 147: 1107-13.

10. Farges O, Fuks D, Boleslawski E, Le Treut YP, Castaing D, Laurent A, et al. Influence of surgical margins on outcome in patients with intrahepatic cholangiocarcinoma: a multicenter study by the AFC-IHCC-2009 study group. Ann Surg. 2011; 254: 824-29; discussion 30.

11. Conci S, Ruzzenente A, Vigano L, Ercolani G, Fontana A, Bagante F, et al. Patterns of Distribution of Hepatic Nodules (Single, Satellites or Multifocal) in Intrahepatic Cholangiocarcinoma: Prognostic Impact After Surgery. Ann Surg Oncol. 2018; 25: 3719-27.

12. Spolverato G, Kim Y, Alexandrescu S, Popescu I, Marques HP, Aldrighetti L, et al. Is Hepatic Resection for Large or Multifocal Intrahepatic Cholangiocarcinoma Justified? Results from a Multi-Institutional Collaboration. Ann Surg Oncol. 2015; 22: 2218-25.

13. Berzigotti A, Reig M, Abraldes JG, Bosch J, Bruix J. Portal hypertension and the outcome of surgery for hepatocellular carcinoma in compensated cirrhosis: a systematic review and meta-analysis. Hepatology. 2015; 61: 526-36.

14. Pugh RN, Murray-Lyon IM, Dawson JL, Pietroni MC, Williams R. Transection of the oesophagus for bleeding oesophageal varices. Br J Surg. 1973; 60: 646-9.

15. Sakamoto Y, Kokudo N, Matsuyama Y, Sakamoto M, Izumi N, Kadoya M, et al. Proposal of a new staging system for intrahepatic cholangiocarcinoma: Analysis of surgical patients from a nationwide survey of the Liver Cancer Study Group of Japan. Cancer. 2016; 122: 61-70.

16. Amin MB, Greene FL, Edge SB, Compton CC, Gershenwald JE, Brookland RK, et al. The Eighth Edition AJCC Cancer Staging Manual: Continuing to build a bridge from a population-based to a more "personalized" approach to cancer staging. CA Cancer J Clin. 2017; 67: 93-9.

17. Uenishi T, Ariizumi S, Aoki T, Ebata T, Ohtsuka M, Tanaka E, et al. Proposal of a new staging system for mass-forming intrahepatic cholangiocarcinoma: a multicenter analysis by the Study Group for Hepatic Surgery of the Japanese Society of Hepato-Biliary-Pancreatic Surgery. J Hepatobiliary Pancreat Sci. 2014; 21: 499-508.

18. Jiang $\mathrm{W}$, Zeng ZC, Tang ZY, Fan J, Sun HC, Zhou J, et al. A prognostic scoring system based on clinical features of intrahepatic cholangiocarcinoma: the Fudan score. Ann Oncol. 2011; 22: 1644-52.

19. Zhou H, Jiang X, Li Q, Hu J, Zhong Z, Wang H, et al. A simple and effective prognostic staging system based on clinicopathologic features of intrahepatic cholangiocarcinoma. Am J Cancer Res. 2015; 5: 1831-43.

20. Ganz P, Heidecker B, Hveem K, Jonasson C, Kato S, Segal MR, et al. Development and Validation of a Protein-Based Risk Score for Cardiovascular Outcomes Among Patients With Stable Coronary Heart Disease. JAMA. 2016; 315: 2532-41.

21. Wu ZF, Wu XY, Zhu N, Xu Z, Li WS, Zhang HB, et al. Prognosis after resection for hepatitis B virus-associated intrahepatic cholangiocarcinoma. World J Gastroenterol. 2015; 21: 935-43.

22. Li YY, Li H, Lv P, Liu G, Li XR, Tian BN, et al. Prognostic value of cirrhosis for intrahepatic cholangiocarcinoma after surgical treatment. J Gastrointest Surg. 2011; 15: 608-13.

23. Ariizumi S, Kotera Y, Takahashi Y, Katagiri S, Chen IP, Ota T, et al. Mass-forming intrahepatic cholangiocarcinoma with marked enhancement on arterial-phase computed tomography reflects favorable surgical outcomes. J Surg Oncol. 2011; 104: 130-9. 
24. Zhou HB, Wang H, Li YQ, Li SX, Wang H, Zhou DX, et al. Hepatitis B virus infection: a favorable prognostic factor for intrahepatic cholangiocarcinoma after resection. World J Gastroenterol. 2011; 17: 1292-303.

25. Zhang L, Cai JQ, Zhao JJ, Bi XY, Tan XG, Yan T, et al. Impact of hepatitis B virus infection on outcome following resection for intrahepatic cholangiocarcinoma. J Surg Oncol. 2010; 101: 233-8.

26. Luo X, Yuan L, Wang $Y$, Ge R, Sun $Y$, Wei G. Survival outcomes and prognostic factors of surgical therapy for all potentially resectable intrahepatic cholangiocarcinoma: a large single-center cohort study. J Gastrointest Surg. 2014; 18: 562-72.

27. Ishikawa K, Sasaki A, Haraguchi N, Yoshikawa Y, Mori M. A case of an alpha-fetoprotein-producing intrahepatic cholangiocarcinoma suggests probable cancer stem cell origin. Oncologist. 2007; 12: 320-4.

28. Ariizumi $S$, Yamamoto $M$. Intrahepatic cholangiocarcinoma and cholangiolocellular carcinoma in cirrhosis and chronic viral hepatitis. Surg Today. 2015; 45: 682-7.

29. Li H, Li J, Wang J, Liu H, Cai B, Wang G, et al. Assessment of Liver Function for Evaluation of Long-Term Outcomes of Intrahepatic Cholangiocarcinoma: A Multi-Institutional Analysis of 620 Patients. Front Oncol. 2020; 10: 525.

30. Akgul O, Bagante F, Olsen G, Cloyd JM, Weiss M, Merath K, et al. Preoperative prognostic nutritional index predicts survival of patients with intrahepatic cholangiocarcinoma after curative resection. J Surg Oncol. 2018; 118: $422-30$.

31. Sasaki K, Margonis GA, Andreatos N, Bagante F, Weiss M, Barbon C, et al. Preoperative Risk Score and Prediction of Long-Term Outcomes after Hepatectomy for Intrahepatic Cholangiocarcinoma. J Am Coll Surg. 2018; 226: 393-403.

32. Tsilimigras DI, Mehta R, Aldrighetti L, Poultsides GA, Maithel SK, Martel G, et al. Development and Validation of a Laboratory Risk Score (LabScore) to Predict Outcomes after Resection for Intrahepatic Cholangiocarcinoma. J Am Coll Surg. 2020; 230: 381-91 e2.

33. Yamamoto Y, Sugiura T, Todaka A, Okamura Y, Ito T, Ashida R, et al. Surgical Indication for Advanced Intrahepatic Cholangiocarcinoma According to the Optimal Preoperative Carbohydrate Antigen 19-9 Cutoff Value. World J Surg. 2018; 42: 3331-40.

34. He C, Zhang Y, Song Y, Wang J, Xing K, Lin X, et al. Preoperative CEA levels are supplementary to CA19-9 levels in predicting prognosis in patients with resectable intrahepatic cholangiocarcinoma. J Cancer. 2018; 9: 3117-28.

35. Pawlik TM, Delman KA, Vauthey JN, Nagorney DM, Ng IO, Ikai I, et al. Tumor size predicts vascular invasion and histologic grade: Implications for selection of surgical treatment for hepatocellular carcinoma. Liver Transpl. 2005; 11: 1086-92.

36. Addeo P, Jedidi I, Locicero A, Faitot F, Oncioiu C, Onea A, et al. Prognostic Impact of Tumor Multinodularity in Intrahepatic Cholangiocarcinoma. J Gastrointest Surg. 2019; 23: 1801-9.

37. Spolverato G, Ejaz A, Kim Y, Sotiropoulos GC, Pau A, Alexandrescu S, et al. Tumor size predicts vascular invasion and histologic grade among patients undergoing resection of intrahepatic cholangiocarcinoma. J Gastrointest Surg. 2014; 18: 1284-91.

38. Lu J, Li B, Li FY, Ye H, Xiong XZ, Cheng NS. Long-term outcome and prognostic factors of intrahepatic cholangiocarcinoma involving the hepatic hilus versus hilar cholangiocarcinoma after curative-intent resection: Should they be recognized as perihilar cholangiocarcinoma or differentiated? Eur J Surg Oncol. 2019; 45: 2173-9.

39. Tarchi P, Tabrizian P, Prigoff J, Schwartz M. Outcomes of resection for solitary $</=5 \mathrm{~cm}$ intrahepatic cholangiocarcinoma. Surgery. 2018; 163: 698-702.

40. Zhang XF, Beal EW, Bagante F, Chakedis J, Weiss M, Popescu I, et al. Early versus late recurrence of intrahepatic cholangiocarcinoma after resection with curative intent. Br J Surg. 2018; 105: 848-56.

41. Durand F, Valla D. Assessment of prognosis of cirrhosis. Semin Liver Dis. 2008; 28: 110-22. 